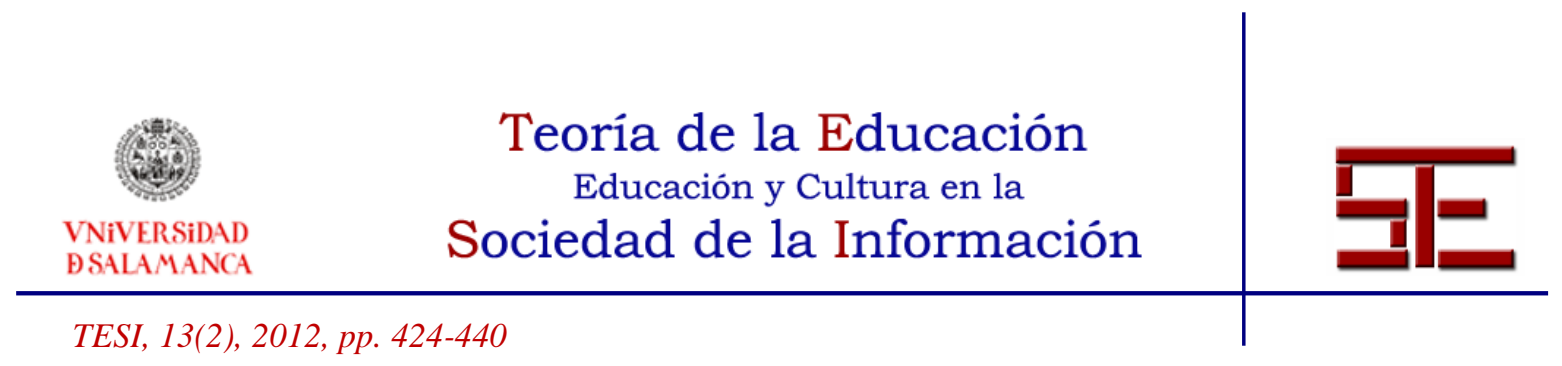

\title{
LOS ESTUDIANTES UNIVERSITARIOS DEL SIGLO XXI EN MÉXICO: DE LA PASIVIDAD A LA AUTONOMÍA Y AL PENSAMIENTO CRÍTICO
}

Resumen: El siglo XXI ha traído consigo una enorme cantidad de transformaciones en muchas esferas de la vida social, educativa, tecnológica, científica y familiar. Estas transformaciones han tocado de manera particular el ámbito educativo, con reformas en sus modelos educacionales y con ello los estudiantes universitarios hacen una ruptura con paradigmas de corte positivista en donde el alumno era visto como una fuente restringida por los arreglos contingenciales del profesor programador, los cuales se establecen incluso antes de la situación instruccional (Hernández Rojas, 1998). Aquel sujeto cuyo desempeño y aprendizaje escolar podía ser arreglado desde el exterior, se encuentra en un proceso de emancipación y toma conciencia ahora participa activamente en su propio aprendizaje. Muchas universidades en México trabajan para dar un giro decisivo proporcionando autonomía en sus estudiantes, mediante modelos como el constructivista, al mismo tiempo que se requiere tengan un pensamiento crítico, pero; esa autonomía no es auténtica, los estudiantes se subsumen al currículo de su institución y generan aprendizajes que se limitan a lo que se les evaluará, aun así el estudiante es reificado.

Palabras clave: Palabras clave: autonomía; aprendizaje; pensamiento crítico.

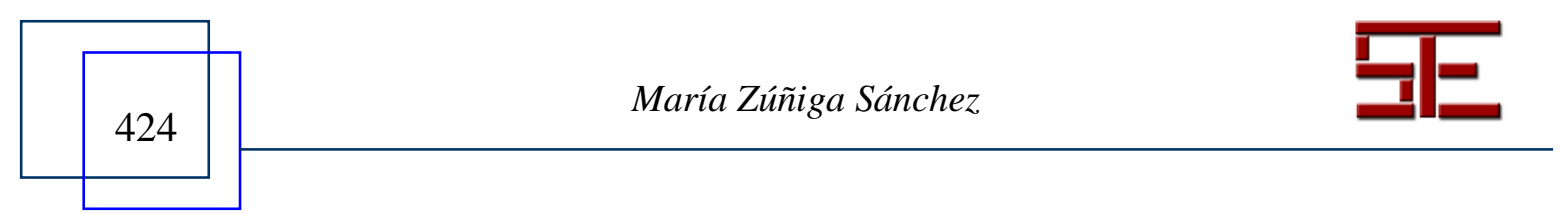




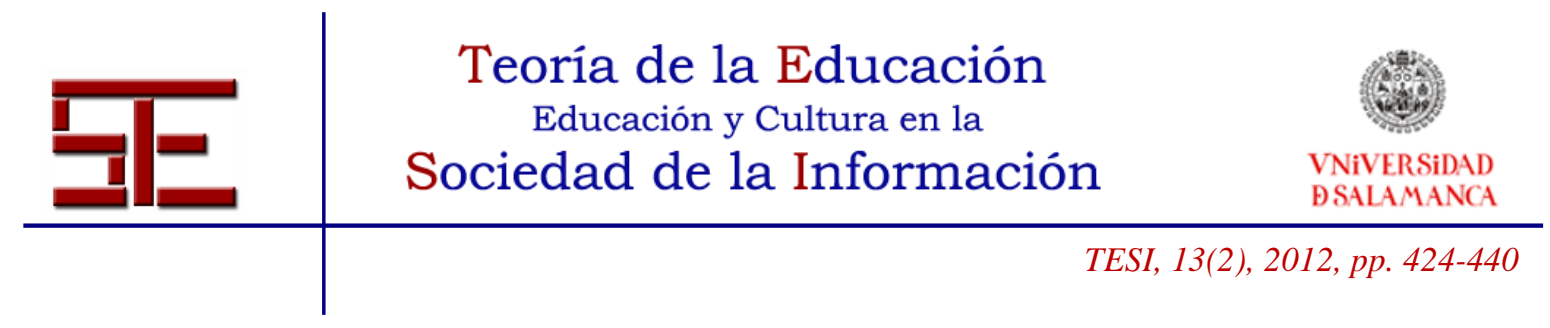

\title{
THE STUDENTS OF XXI CENTURY IN MEXICO: FROM THE PASSIVITY TO AUTONOMY AND CRITICAL THINKING.
}

\begin{abstract}
XXI century has brought an enormous amount of transformations in many spheres of social, educative, technological, scientific and familiar life. These transformations have touched on a particular way the educative ambit, with reforms in their educational models and thus college students make a break with positivist paradigms in which student was seen as a restricted supply by the professor programmer contingencial arrangements, which are set even before the instructional situation (Hernández Rojas, 1998). That subject, whose school performance and learning could be fixed from the outside, is in a process of emancipation and awareness now actively involved in its own learning. Many universities in Mexico are working to give a decisive turn providing autonomy in their students, by models such as constructivism, at the same time they required critical thinking, but this autonomy is not authentic, students are subsumed into their institution curriculum and they generate learning that is limited to what will be assess, yet student is reified.
\end{abstract}

Keywords: Key words: autonomy; learning; critical thinking.

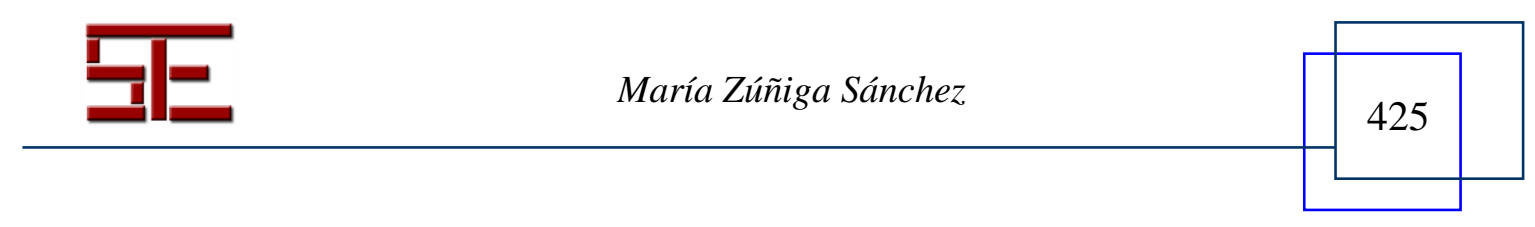




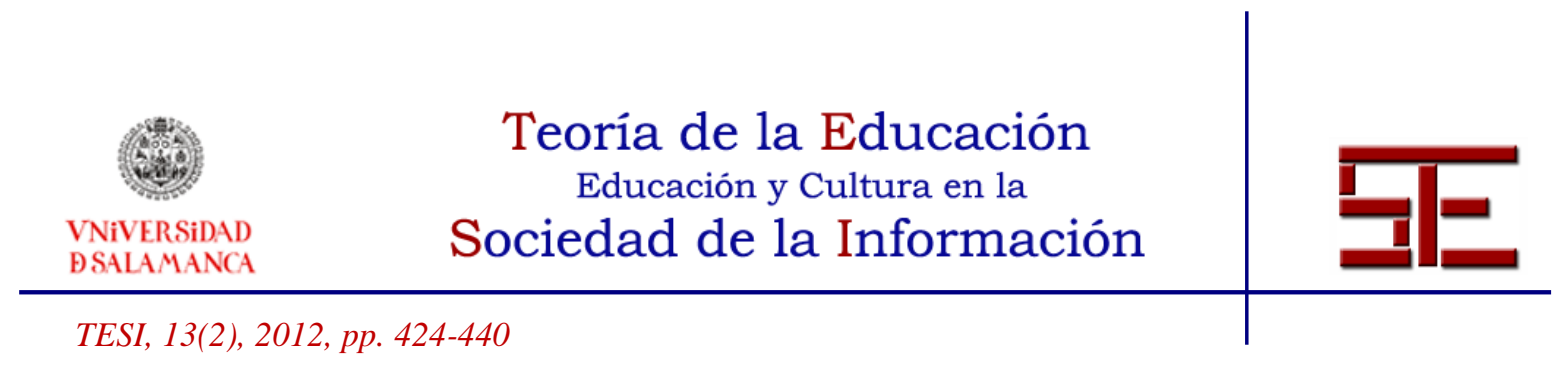

\section{LOS ESTUDIANTES UNIVERSITARIOS DEL SIGLO XXI EN MÉXICO: DE LA PASIVIDAD A LA AUTONOMÍA Y AL PENSAMIENTO CRÍTICO}

Fecha de recepción: 20/09/2011; fecha de aceptación: 20/10/2011; fecha de publicación: 26/07/2012

María Zuñiga Sánchez

mzsegz@hotmail.com

Universidad Autónoma del Estado de Hidalgo

\section{INTRODUCCIÓN}

Este trabajo tiene como propósito hacer una reflexión teórica sobre los estudiantes de algunas universidades mexicanas que buscan autonomía en sus propios aprendizajes, se orientan hacia la práctica del pensamiento crítico, esto, gracias a las transformaciones que la era globalizadora trae consigo en los ámbitos social, cultural, educativo, tecnológico, científico y familiar. Se toman como ejes de análisis las reformas en los modelos educacionales, la ruptura con el modelo positivista, la toma de conciencia, autonomía y el pensamiento crítico en los estudiantes universitarios, para finalizar se llega a conclusiones interesantes sobre el estudiante universitario, sus potencialidades de autonomía y pensamiento crítico, así como las limitaciones. Es importante aclarar que la autonomía se entiende como la condición de quien tiene en sí mismo la norma de su propio comportamiento (Galimberti, 2006). Agregaría que no únicamente el control del comportamiento, sino de sus pensamientos y emociones.

\section{UN NUEVO SIGLO: GRANDES TRANSFORMACIONES}

La era globalizadora ha alcanzado a muchos rincones del mundo, el caso de México no es la excepción. Uno de los principales retos que enfrentarán las Instituciones de Educación Superior (IES) será dar respuestas concretas a una sociedad, más allá de considerarla como una simple entidad abstracta con capacidad de consumo (Maldonado, 2000). Tomando en cuenta que la función de las IES es la generación y aplicación del conocimiento que sustente el orden social y el crecimiento económico del país por medio de la producción de saberes científicos, tecnológicos y culturales, así, se genera un talento humano en los estudiantes que influye de manera positiva en la dinámica de la economía y de la competencia con otros países.

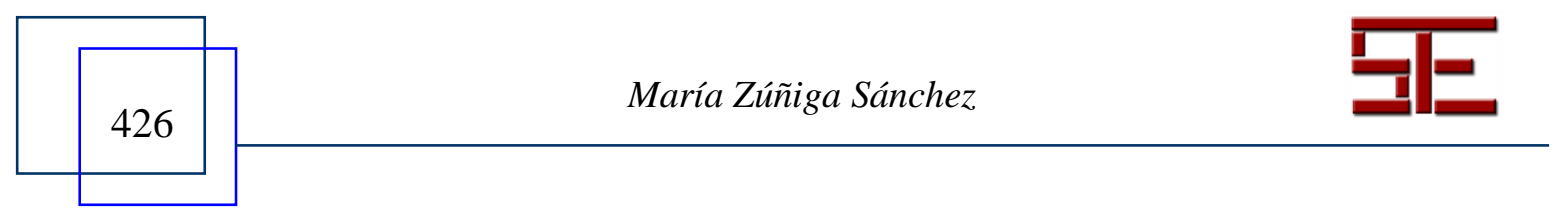




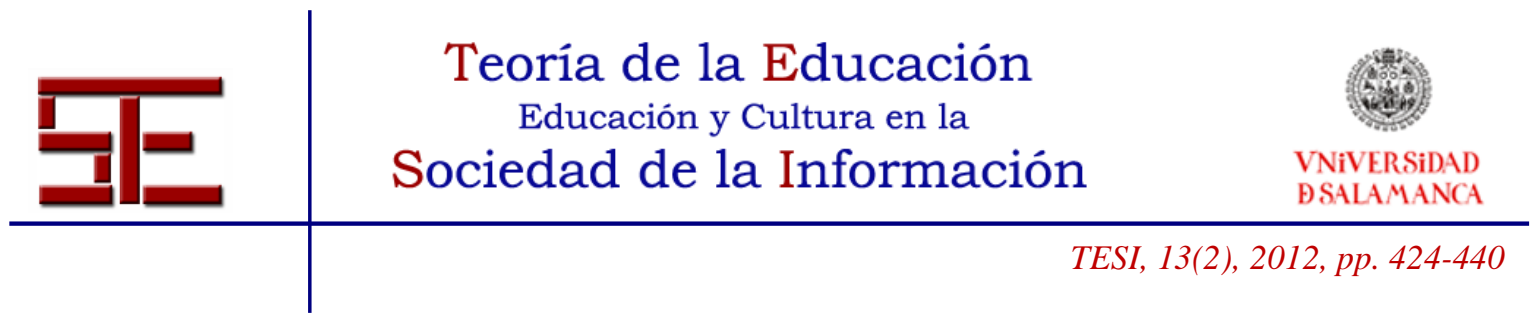

Las tres últimas décadas del siglo XX permiten identificar ciertas fases en la educación superior en México. Luengo (2003) nos da a conocer una etapa de expansión en los años setentas, donde la política gubernamental puso énfasis en los procesos cuantitativos más que en la calidad de los resultados de estos procesos, pues a finales de esta década se dio el menor nivel de eficiencia terminal, tendencia que frenó la expansión.

Otra fase fue de desaceleración, caracterizada por el freno al crecimiento del sistema educativo superior, influida por la crisis económica en 1982, la cual redujo los recursos públicos destinados a la educación superior, en este periodo se acentúa la expansión de la educación superior privada, la crisis económica afectó a todos los campos de la sociedad mexicana incluida la educación, en la educación superior se agudizaron tensiones que llegaron a paralizar algunas instituciones como la Universidad Autónoma de México UNAM, pues cambiaron las relaciones entre el gobierno y las universidades, a mediados de los años ochenta el rector Jorge Carpizo intento varias reformas educativas, posteriormente existió un nuevo marco de relaciones entre el Estado y las universidades.

La fase evaluadora es la última, se deja ver la supervisión estatal y la asignación de recursos, ocurre en los años noventa, donde las relaciones entre Estado y universidades mejoraron, creando un nuevo dispositivo de vigilancia a distancia y la autonomía regulada del sistema educativo superior, lo cual ha generado un amplio repertorio de instrumentos de evaluación tanto nacionales como internacionales en sus programas académicos, acreditación de programas académicos de pos grado, evaluación de proyectos para asignar recursos económicos, exámenes generales de alumnos, evaluación internacional, entre otros.

Con esto en mente, podemos darnos de cuenta que la educación superior en México ha pasado por etapas de conflicto, y gracias a esto las universidades han generado oportunidades para la mejora, pues muchas de las que se mencionan en este trabajo han luchado para reformar sus modelos educativos para dar solución a los problemas de la sociedad en la vida actual.

La educación tiene la misión de permitir a todos sin excepción hacer fructificar todos sus talentos y todas sus capacidades de creación, lo que implica que cada uno pueda responsabilizarse de sí mismo y realizar su proyecto personal (Delors, 1999, 13).

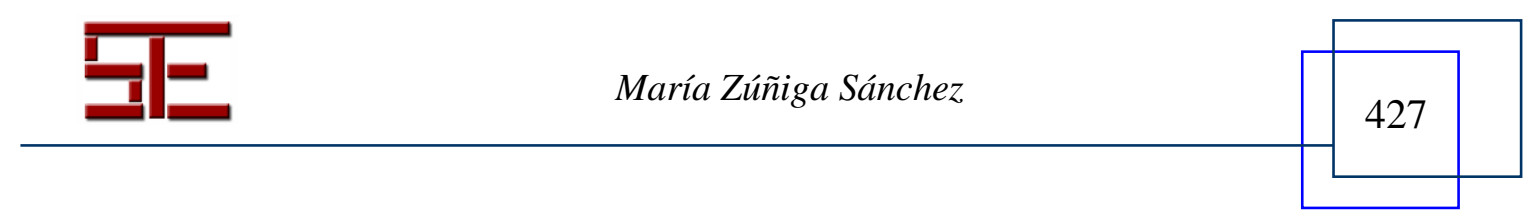




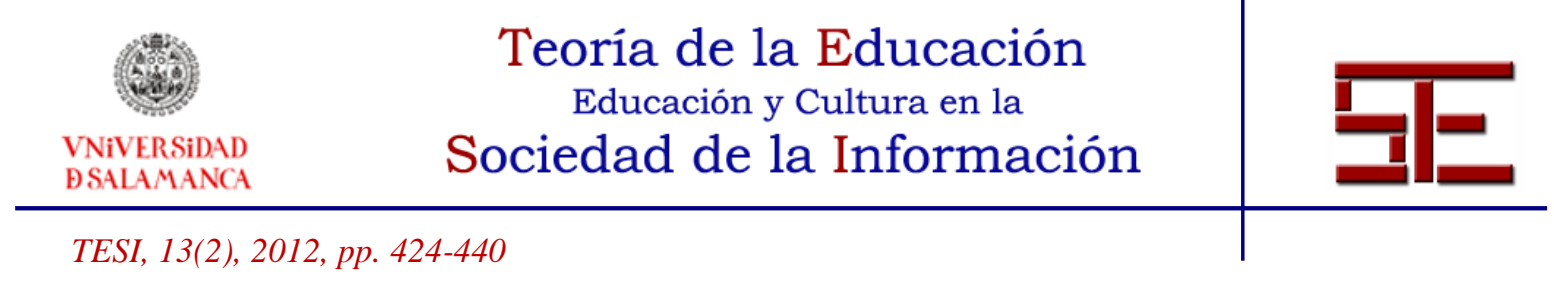

Este mismo autor recomienda que se tomen en cuenta los 4 pilares de la educación en todos los niveles, estos son:

1.- Aprender a conocer combinando una cultura general para profundizar los conocimientos en un pequeño número de materias, esto supone aprender a aprender a lo largo de toda la vida.

2.-Aprender a hacer para adquirir no sólo una calificación profesional, sino una competencia que capacite al individuo a hacer frente a situaciones y trabajar en equipo. También aprender a hacer en el marco de experiencias sociales o nacionales.

3.-Aprender a vivir juntos desarrollando la comprensión del otro y las formas de interdependencia, trabajar proyectos comunes y resolver los conflictos con los valores de pluralismo, comprensión mutua y paz.

4.-Aprender a ser para que la propia personalidad esté en condiciones de obrar con creciente capacidad de autonomía, de juicio y de responsabilidad personal.

En este trabajo nos centraremos en Aprender a ser, pues es un eje rector de toda universidad, ya que invita al estudiante y a los docentes a obrar con autonomía, juicio y responsabilidad, cabe mencionar que el ser autónomo implica tomar en serio un conjunto de responsabilidades como comprometerse con uno mismo, disciplinarse y generar un gran discernimiento para la adecuada toma de decisiones en la universidad y fuera de ella.

\section{REFORMAS EN MODELOS EDUCACIONALES DE ALGUNAS UNIVERSIDADES MEXICANAS}

En la actualidad las presiones para reformar las instituciones de Educación Superior latinoamericanas, a diferencia del pasado, provienen más del "exterior" que del “interior” de las instituciones (Brunner, 1999, citado por Alcántara, 2006).

La universalización de la escolarización y la oferta pertinente de educación no bastan por sí solas para garantizar la eficacia y el éxito, estos dependen también de la calidad (UNESCO, 2005) como el número de estudiantes por docentes, la formación del profesorado, la calidad en infraestructuras, el material puesto a disposición de los estudiantes y docentes. Este organismo internacional refiere que aunque algunos países ricos han logrado universalizar la educación aún existe cerca del 25 por ciento de la población que no cuentan con las competencias y aptitudes necesarias para participar plenamente en la vida social y laboral. Es importante considerar que estos factores

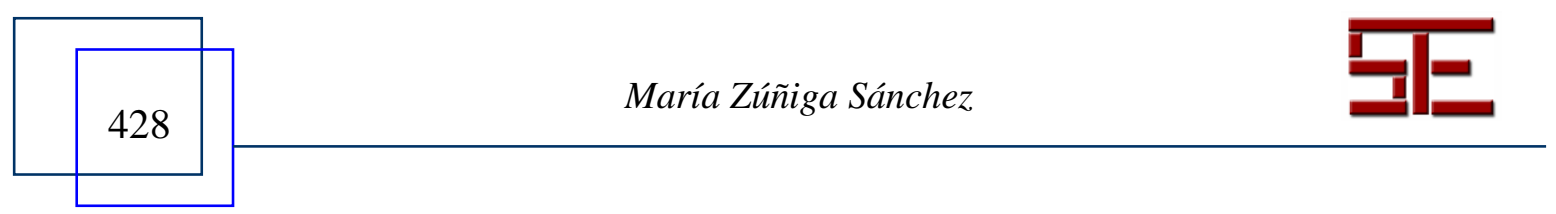




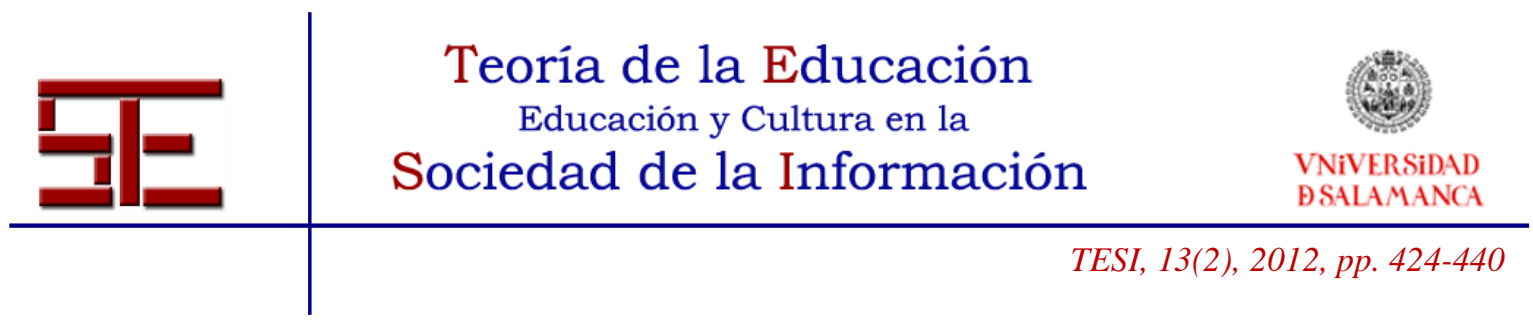

señalan una línea divisoria entre el éxito y el fracaso en la vida laboral que influye en la economía de la sociedad mexicana, pero no sólo en la economía, pues también las otras esferas de la vida se ven trastocadas.

\section{RUPTURA CON EL PARADIGMA POSITIVISTA DESDE EL PUNTO DE VISTA DEL APRENDIZAJE: EL ROL DEL PROFESOR Y DEL ESTUDIANTE}

El paradigma positivista tuvo su auge en las primeras décadas del siglo XX. Así que, Hernández R. (2006) comenta que entre la década de 1940 y 1960, el conductismo skinneriano se desarrolló y protagonizó la escuela académica, como sabemos, el conductismo se inserta en una tradición filosófica empirista de la cual heredó tres características: el ambientalismo (considera que el medio ambiente físico y social determina la forma de comportamiento de los organismos), el asociacionismo (causalidad) y el anticonstructivismo (no toma en cuenta las estructuras internas de los sujetos, es decir, las series de procesos mentales organizativos).

Los conocimientos de los sujetos son considerados desde este enfoque como una serie de asociaciones entre estímulos y respuestas.

En comunión con Skinner varios investigadores realizaron experimentos sobre la conducta con animales para buscar leyes que dieran cuenta del comportamiento humano.

Esos trabajos influyeron en la manera de educar a los estudiantes en las escuelas, pues este paradigma concebía al alumno como: un sujeto cuyo desempeño y aprendizaje escolar podían ser arreglados o rearreglados desde el exterior (Hernández, 2006), es decir, su situación instrucciones, los métodos, los contenidos, etcétera. El estudiante era un sujeto pasivo, un objeto moldeable por los arreglos contingenciales que realizaba el maestro, pues el trabajo de éste consistía en desarrollar una serie de arreglos de contingencia de reforzamientos y control de estímulos para enseñar. Este modelo se adoptó en México por muchas décadas.

Por fortuna actualmente son pocas las escuelas que trabajan con este paradigma, especialmente en educación básica, pues los organismos internacionales han realizado recomendaciones para que se acentúe la autonomía en los estudiantes y se promueva el pensamiento crítico.

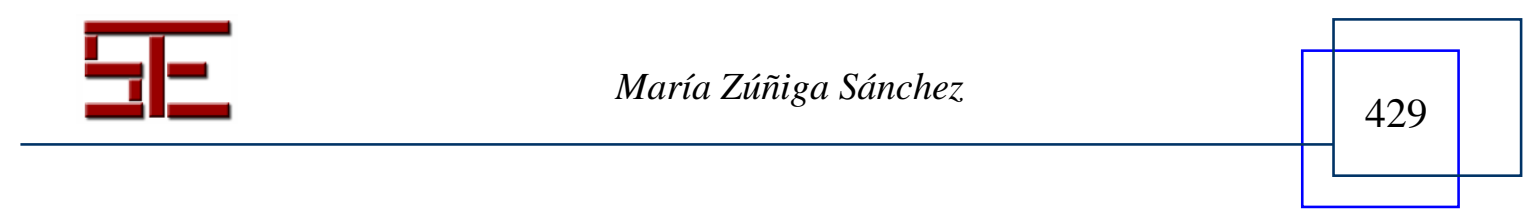




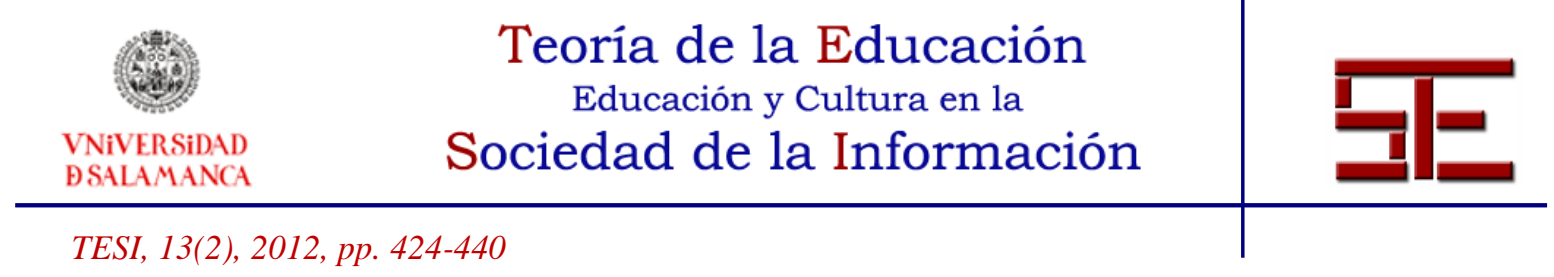

Es así que, algunas universidades han adoptado el paradigma constructivista, como es el caso de la Universidad Autónoma del Estado de Hidalgo que en su nuevo Modelo Educativo promueve una relación bidireccional entre estudiante y profesor.

Los estudiantes han dejado de ser sujetos pasivos y la relación profesor-estudiante ha dejado de ser unidireccional.

El Modelo constructivista adoptado por la UAEH concibe al estudiante como constructor activo de su propio conocimiento y el reconstructor de los distintos contenidos escolares a los que se enfrenta, el profesor promueve el desarrollo psicológico y la autonomía de los estudiantes.

Basañez (2011) menciona que la familia y la escuela lamentablemente han generado personas disciplinadas, pero inhiben creatividad e innovación. Sugiere que el profesor deje de enfatizar el "pórtate bien" por el "explora, arriesga, busca y propón".

\section{TOMA DE CONCIENCIA: EMANCIPACIÓN}

Alienación es un término que utilizó Marx para dar cuenta de la falta de conciencia del grupo de campesinos que eran explotados por los burgueses posteriormente a la revolución industrial, muchos autores abordan este tema con palabras que parecieran ser sinónimos, como enajenación o ser en sí.

El ser en sí, señala Sartre (citado por Gutiérrez, 2007), es el objeto, carece de conciencia, de movimiento, de relaciones, en oposición está el para sí; en cual es consciente, sujeto, es lo típicamente humano.

Es así que los universitarios del siglo XXI en México muestran gran interés por tomar conciencia de sus aprendizajes, los docentes coadyuvan a que se desarrolle el pensamiento crítico para resolver problemas y tomar decisiones apropiadas para cada situación dentro y fuera de la universidad. Pues la sociedad globalizada demanda profesionales con autonomía y pensamiento crítico.

Permítanme hacer una analogía sobre los universitarios del siglo XXI con el "Mito de la caverna" que narra Platón en el libro VII de la República, donde habla sobre el mundo de las ideas y el Mundo sensible:

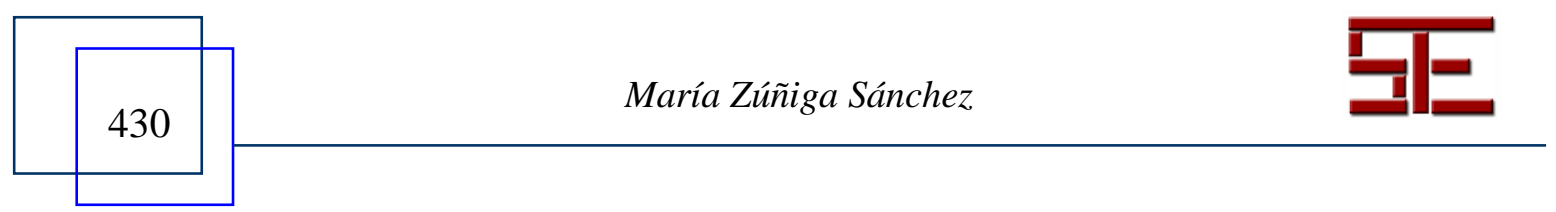




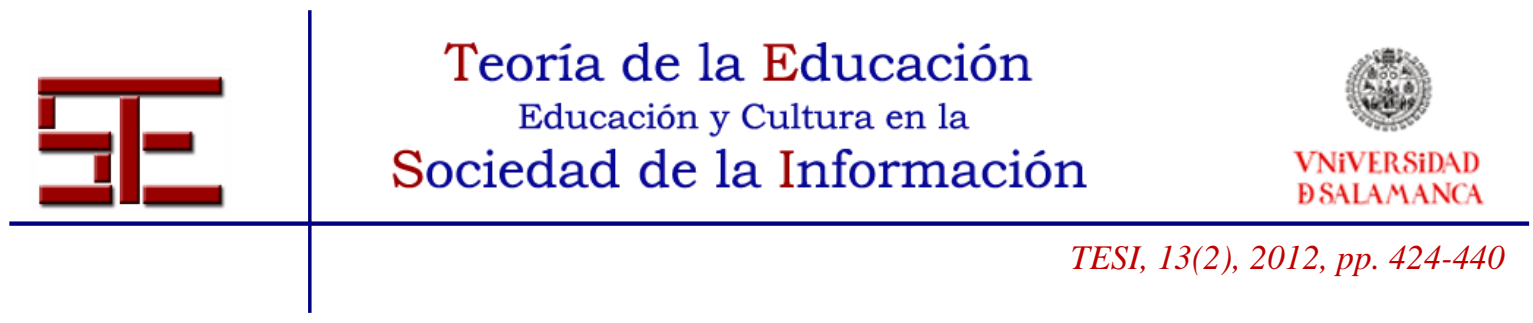

"En una caverna oscura están varios prisioneros, atados desde la infancia; no pueden ver la luz del día, ni los objetos y personas del exterior. Sólo captan unas sombras que se proyectan en el fondo de la caverna; afuera hay un camino y más lejos un fuego, que origina esas sombras. Uno de los prisioneros escapa y, al principio, queda deslumbrado por la luz del día. Poco a poco se acostumbra a ver y a mirar, maravillado, los objetos y personas que antes ni sospechaba, vuelve con sus compañeros, pero éstos no le creen lo que narra, están convencidos de que la única realidad es lo que ven en el fondo de la caverna" (Gutiérrez, 2007, 45-46).

De tal mito, Platón deducía que los prisioneros representan a la mayor parte de la humanidad, la caverna es el mundo sensible, en el que habitamos; el exterior es el mundo intelectual de las ideas; el fuego representa el bien o la luz.

En el mundo sensible captamos solo las sombras de la verdadera y perfecta realidad, que está en un mundo aparte, invisible a las captaciones cotidianas (Gutiérrez, 2007, 46).

Con base en todo lo anterior, se puede comparar al estudiante autónomo y con pensamiento crítico que se ha emancipado con el filósofo que tiene la intuición de las ideas, la palabra filósofo deriva de dos vocablos griegos: Filos que significa Amor y Sophia se refiere a sabiduría, el filósofo entonces, es un ser que ama la sabiduría, pero, en el mito, su enseñanza es despreciada por los demás prisioneros ante sus narraciones acerca del mundo superior, quedándose estos últimos en el plano de la doxa, el filósofo en un primer momento "no puede ver claramente", no obstante, poco a poco ese amor a la sabiduría lo conquista. El estudiante autónomo y con pensamiento crítico pasa de la doxa al plano de la episteme, pues sabe que es responsable de cuanto quiere aprender.

\section{AUTONOMÍA EN LA CONSTRUCCIÓN DEL APRENDIZAJE EN LOS ESTUDIANTES: ¿EXISTE LA AUTÉNTICA AUTONOMÍA EN LOS ESTUDIANTES?}

Kammi (1982, citado por Hernández) define la autonomía como ser capaz de pensar críticamente, por uno mismo, en el terreno moral e intelectual, tomando en cuenta y coordinando diversos puntos de vista.

¿Desde qué etapa de la vida podemos ser autónomos?

La perspectiva psicológica de Erik Erikson (1963, citado por Frager, y Fadiman, 2009), señala que, pasamos por 8 etapas de desarrollo humano, al que llamó epigenético, en el

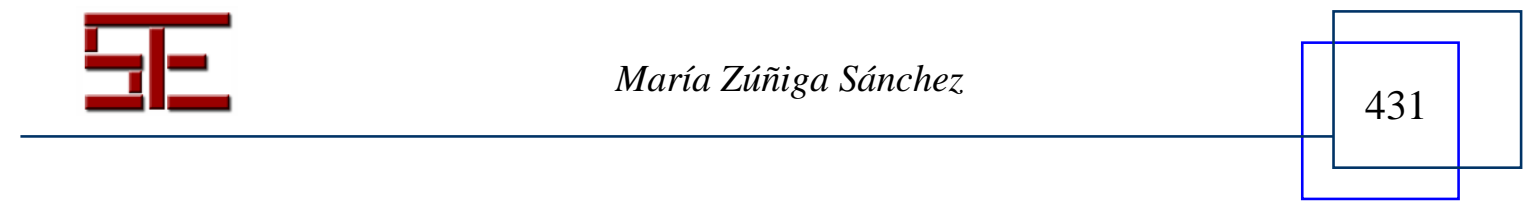




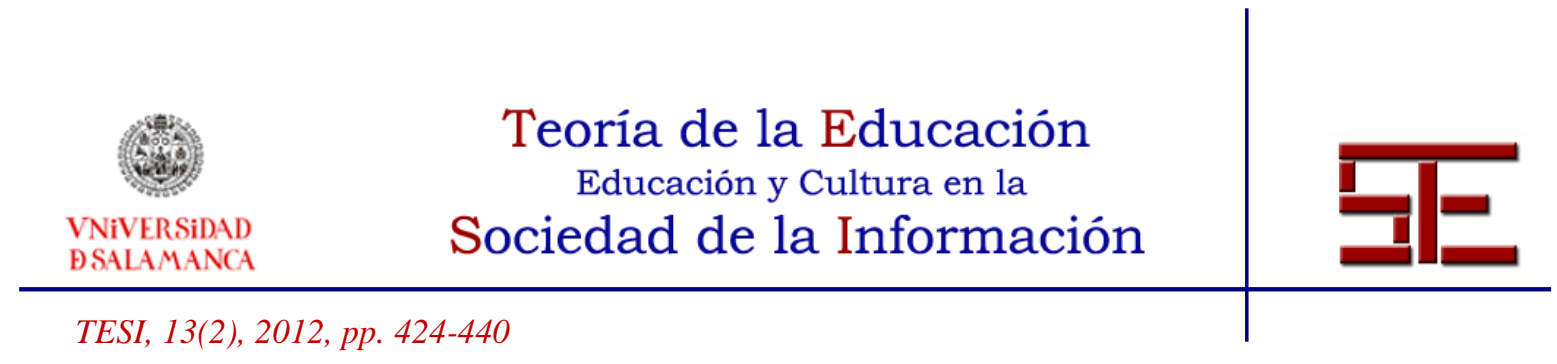

cual detalla el ciclo de la vida desde la infancia hasta la madurez y la vejez, su teoría tiene dos premisas básicas:

1.- La personalidad humana se desarrolla de acuerdo con los pasos determinados por la capacidad de progresar, de saber y de relacionarse con una esfera social.

2.- La sociedad está constituida de manera que cumpla y estimule la sucesión de potencialidades para la interacción, alentar la secuencia y el ritmo adecuado de desarrollo.

Con esto en mente, Erikson expone 8 etapas de desarrollo, en cada una se desarrolla un momento crítico que fortalece una virtud ${ }^{1}$ psicológica. En la segunda etapa, el momento crítico es una lucha entre autonomía o vergüenza o duda, ocurre a temprana edad, en donde el niño adquiere destrezas mentales y físicas como sostenerse y comunicarse de mejor manera, se relaciona con el mundo y comienza a ejercer control sobre sí mismo anunciando una autonomía cada vez mayor. La contraparte de la autonomía en esta etapa es la vergüenza como un sentimiento de que las deficiencias son visibles para los demás, en este sentido, la virtud que se adquiere es la voluntad relacionada con la posibilidad de controlar los propios impulsos con juicio $\mathrm{y}$ discernimiento; es decir, tomar decisiones, la voluntad determina el libre albedrío.

De modo que esta teoría psicológica nos permite visualizar que tenemos la capacidad de ser autónomos desde los primeros años de vida, por supuesto en esta etapa nos referimos únicamente al control de nuestro organismo biológico y gradualmente se va gestando la autonomía intelectual. También la teoría psicológica freudiana señala que la autonomía se inicia con la fase ${ }^{2}$ anal, precisamente con el control de los esfínteres (Galimberti, 2006).

${ }^{1}$ Utiliza el término "virtud" en su viejo sentido, como la virtud de una medicina; se refiere más a las capacidades que a la moral. (Frager, R. y Fadiman J. 2009. pp205).

${ }^{2}$ La teoría psicosexual de Sigmund Freud se compone de 5 fases:

Fase oral, anal, fálica, latencia y genital. En cada fase la libido o impulso se concentra en algún órgano del cuerpo.

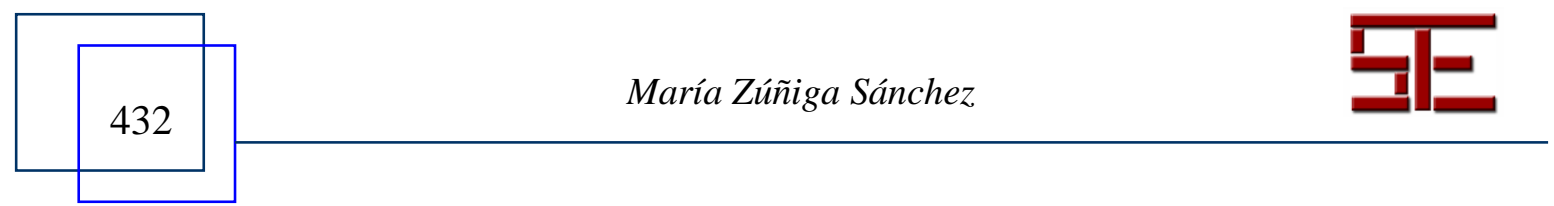




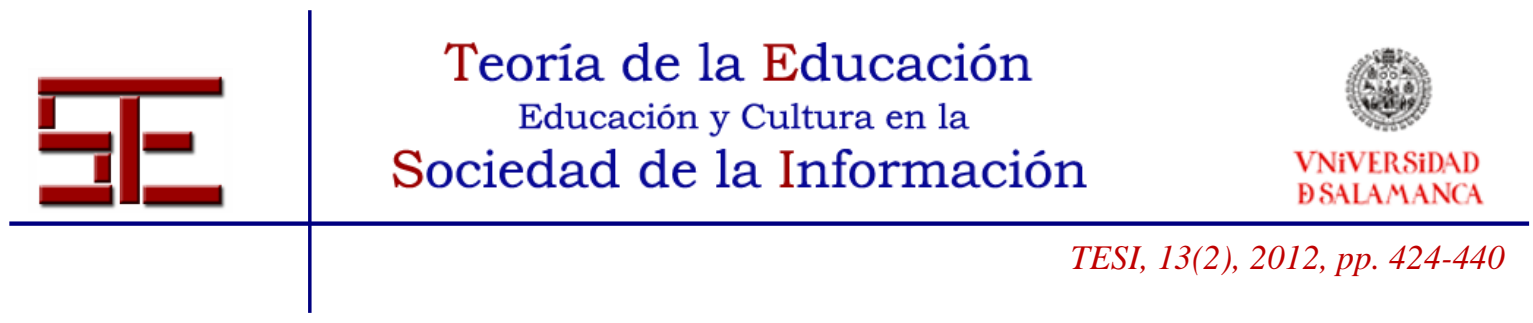

Veamos ahora qué recomendaciones tiene la UNESCO (2005) respecto a la autonomía. Señala que es importante que los ciudadanos expresen sus aspiraciones y opciones en materia de educación. Es decir, este organismo internacional impulsa a los estudiantes universitarios a hablar de sus deseos, anhelos, ambiciones relacionados con su formación académica, gestándose así la autonomía. Cabe señalar que a lo largo de la historia algunos estudiantes han mostrado autonomía en sus aprendizajes, sin embargo es en este siglo que se puede hablar del ejercicio de la autonomía con mayor profundidad.

De esta manera, Amartya Sen (citado por la UNESCO, 2005) confirma que el desarrollo humano es inconcebible sin la libertad de expresión. Conceder la autonomía a los estudiantes no significa que éste decida ir o no a clase, es así que Saenz; Galán y Luna (2003) del Centro de Estudios Sobre la Universidad, Centro de Innovación y Competitividad de la Universidad Autónoma de Nuevo León, México creen que la asistencia a clases debería dejar de ser obligatoria, pues dependerá del grado de madurez exigido a los alumnos para acceder a la universidad. Tampoco significa que se permita la entrada al libertinaje, de modo tal, que la autonomía del estudiante incide en superar los problemas pedagógicos, para que los estudiantes aprendan por ellos mismos, los docentes deben potenciar su capacidad analítica viendo todas las perspectivas de los fenómenos a estudiar, más que su capacidad memorística. Pues es más fácil para un estudiante comprender una ley si le explicamos su porqué, su cómo y su finalidad.

El Centro de Estudios Sobre la Universidad, Centro de Innovación y Competitividad de la Universidad Autónoma de Nuevo León, México tiene algunas propuestas para desarrollar la capacidad analítica en los estudiantes para que sean autónomos en sus aprendizajes:

- $\quad$ Olvidar el sistema de apuntes o de utilización básica de manuales.

- $\quad$ El profesor aclara conceptos y sirve de guía, pero deja que los estudiantes analicen y aprendan desde la comparación y acumulación de diversos referentes.

- Fomentar el debate entre todos los estudiantes y el profesor.

- Los métodos para evaluar pueden ser trabajos que se relacionan con la evaluación continua y el examen escrito (no memorístico), es decir analítico, como por ejemplo; desarrollar un comentario de un tema.

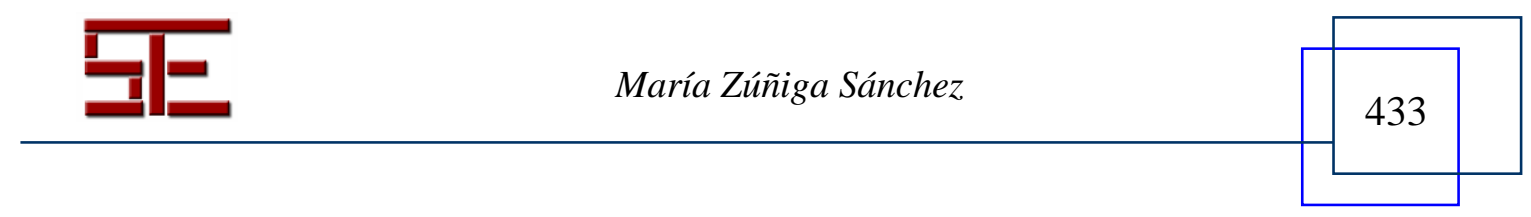




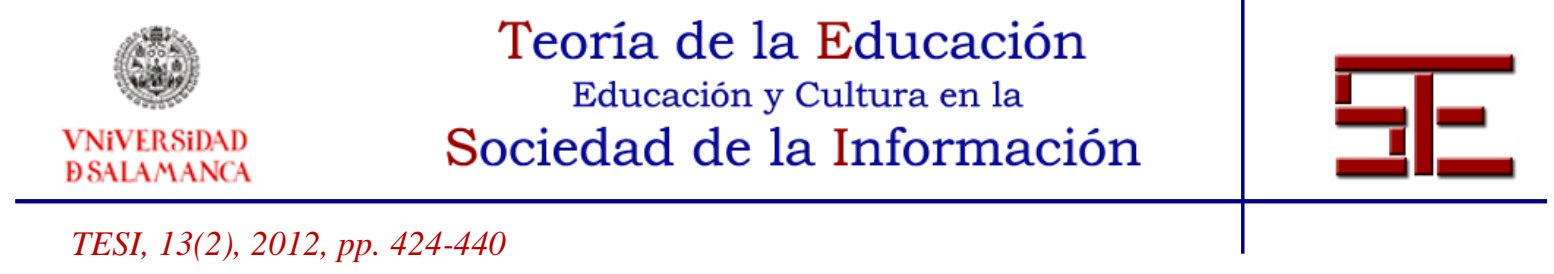

Ciertamente, la autora está de acuerdo con esta postura que se adopta en Nuevo León, no obstante, cabe señalar que es indispensable considerar ciertos valores que permean indiscutiblemente para lograr la autonomía de los estudiantes; estos son el respeto y la tolerancia, en una relación tripartita entre estudiantes-profesor, estudiantes-estudiantes y profesor-estudiantes.

De modo que hablar acerca de la autonomía del estudiante también es hablar de manejo de sí, como una competencia que trata de dotar a los alumnos de las actitudes, habilidades y conocimientos que les permitan buscar el desarrollo profesional y personal a través de la reflexión, el discernimiento y el diálogo que conduzca a un proyecto de vida Autónomo, comprometido y congruente (UIA, 2002, citado por Navarro y Rendón González, 2004). Cabe señalar que los profesores tienen un gran compromiso de promover el autoconocimiento, la autoestima y la asertividad en los estudiantes, de esta manera se puede aprender a ser autónomo.

\section{ESTUDIANTES UNIVERSITARIOS Y EL PENSAMIENTO CRÍTICO.}

¿Qué es el pensamiento crítico? Es una habilidad intelectual compleja que desarrollamos los seres humanos.

El pensamiento es un proceso que tiene lugar en el cerebro de las personas y que depende de factores intrínsecos, algunos fijados por la herencia, otros por el aprendizaje y extrínsecos como la información disponible y el modo en que se acceda a ella (Castellano, M. H. 2007. 18). Cuando se habla de factores intrínsecos se hace referencia a aquellas motivaciones que se centran en la tarea misma y en la satisfacción personal que representa enfrentarla con éxito, por otro lado la motivación extrínseca depende de lo que digan o hagan los demás respecto a la actuación del alumno (Díaz, y Hernández $\mathrm{R}, 2010$ ). Es así que, más adelante hablamos acerca de aquellos factores extrínsecos que en un tiempo moldearon las acciones de los universitarios en México.

De acuerdo con Castellano (2007, 110), los estudiantes con habilidad de pensamiento crítico se caracterizan por tener diferentes disposiciones: Disposición a la osadía mental, hacia la curiosidad intelectual, a clarificar y perseguir la comprensión, Planificar y diseñar estrategias, ser intelectualmente cuidadoso, buscar y evaluar razones y ser meta cognitivo. En el caso de los estudiantes universitarios mexicanos estas disposiciones son evidentes, pero aún más, en los niveles de posgrado, pues se requiere planificar, clarificar y ser meta cognitivos porque realizan investigaciones.






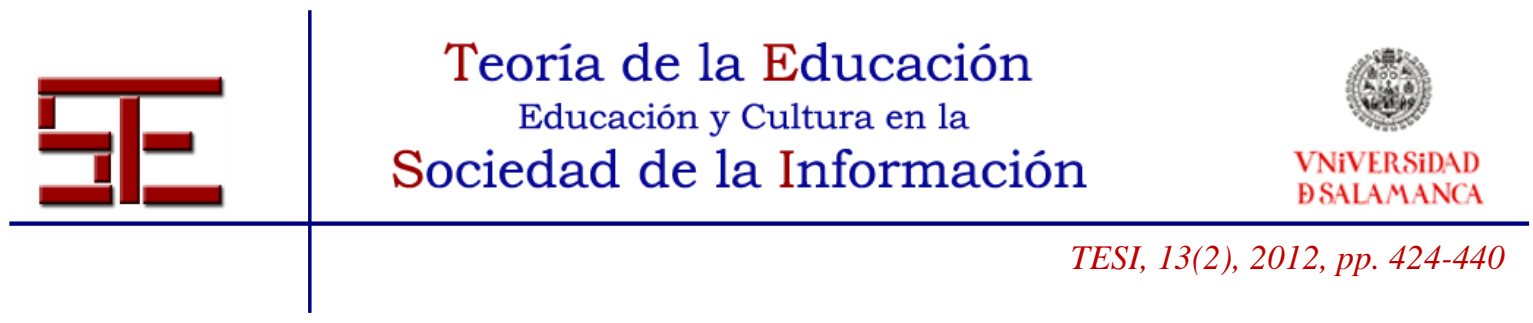

Un ejemplo empírico, es mi grupo de posgrado "Maestría en Ciencias de la Educación" como su nombre lo indica, revisamos varias ciencias enfocadas a la educación y a la investigación, mismas que requieren de la correcta comprensión para poder vincular las teorías a los temas de investigación, teniendo claro que la elección de una teoría o una postura epistemológica es un compromiso para el diseño del proyecto, del instrumento y para la interpretación y análisis de resultados, es así que el estudiante requiere incorporar el pensamiento crítico para lograr la consecución de las demandas pedagógicas que exige el programa de la maestría.

El uso del pensamiento crítico es necesario en todos los niveles educativos, así también, todo ser humano cuenta con la característica de pensamiento crítico que puede desarrollar en diferentes grados.

El autor Rugarcia (1997) señala algunas características importantes del pensamiento crítico:

- $\quad$ El pensamiento crítico se desarrolla, no se aprende.

- $\quad$ No es un conocimiento, es un método basado en un razonamiento cuidadoso en la búsqueda o aplicación del conocimiento.

- $\quad$ Es una habilidad intelectual compleja.

- $\quad$ El pensador crítico requiere ciertas actitudes como: cuestionar, dudar, afán por la verdad y sensibilidad al contexto.

El mismo autor da cuenta de algunas habilidades que se relacionan con el pensamiento crítico: comparar, resumir, observar, clasificar, interpretar, criticar, descubrir suposiciones, colectar y organizar datos y tomar decisiones.

Como mencionamos anteriormente, el crecimiento económico del país no sólo depende de la dinámica de las inversiones, sino también de la productividad de los recursos humanos, que deben estar preparados adecuadamente. Al respecto, el Consejo de Especialistas para la Educación en México (2006) señala que la educación en ciencia y tecnología contribuye no solo a la formación de un pensamiento crítico y abierto, sino también a la mejora de las capacidades para enfrentar los retos de las sociedades modernas.

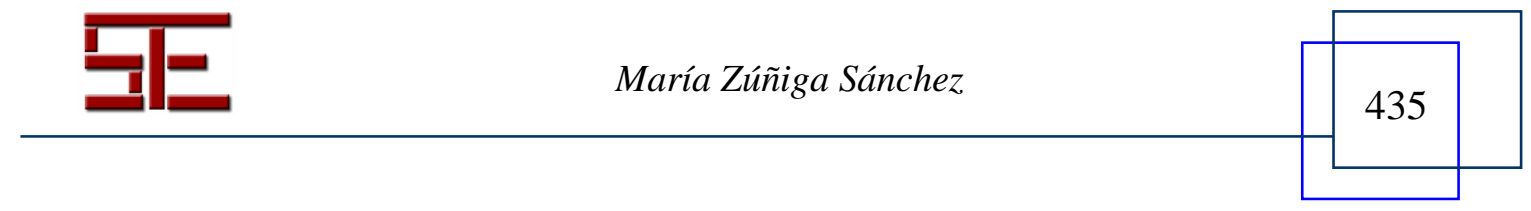




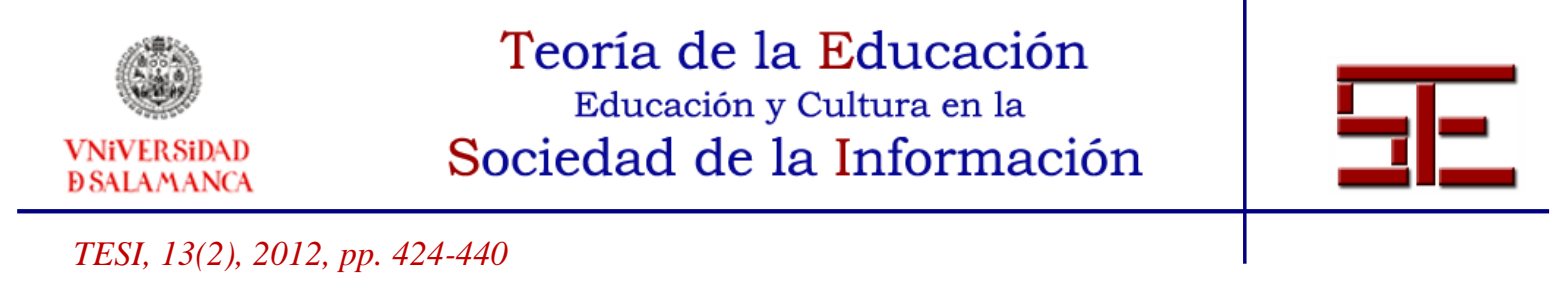

\section{LO EMPÍRICO}

Las universidades mexicanas han realizado cambios en sus modelos educativos, influenciados por los cambios de formas de vida, pues estas instituciones tienen la misión de formar a profesionistas que atiendan las necesidades de su entorno por medio del conocimiento.

La Universidad veracruzana ha tomado en cuenta las recomendaciones de la UNESCO, respecto a las habilidades que deben poseer los egresados; el aprendizaje permanente, el desarrollo autónomo, el trabajo en equipo, la destreza en la solución de problemas, la comprensión de diversas culturas, entre otras.

La Universidad veracruzana UV tiene en su modelo educativo los siguientes objetivos. Objetivo General: Propiciar en los estudiantes de las diversas carreras que oferta la Universidad Veracruzana una formación integral y armónica: intelectual, humana, social y profesional.

Objetivos particulares: Desarrollar en los estudiantes conocimientos, habilidades, destrezas, actitudes y valores necesarios para lograr:

- La apropiación y desarrollo de valores humanos, sociales, culturales, artísticos, institucionales y ambientales.

- Un pensamiento lógico, crítico y creativo.

- El establecimiento de relaciones interpersonales y de grupo con tolerancia y respecto a la diversidad cultural.

- Un óptimo desempeño fundado en conocimientos básicos e inclinación y aptitudes para la auto-formación permanente.

Cabe aclarar que México es un país rico en diversidad cultural, especialmente el estado de Veracruz.

Un proyecto llamado Inter-saberes, implementado por un grupo de investigadores, dio pauta para que la Universidad Veracruzana iniciara un programa intercultural iniciando la institucionalización de la diversidad cultural. Esto permitió la descentralización de la UV a varias regiones pluriétnicas del estado (Dietz y Mateos, 2010). Es interesante señalar que el modelo educativo de esta universidad promueve el pensamiento crítico,

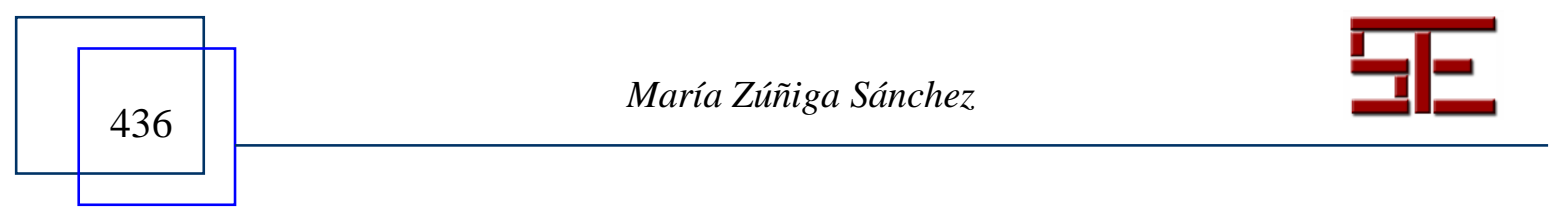




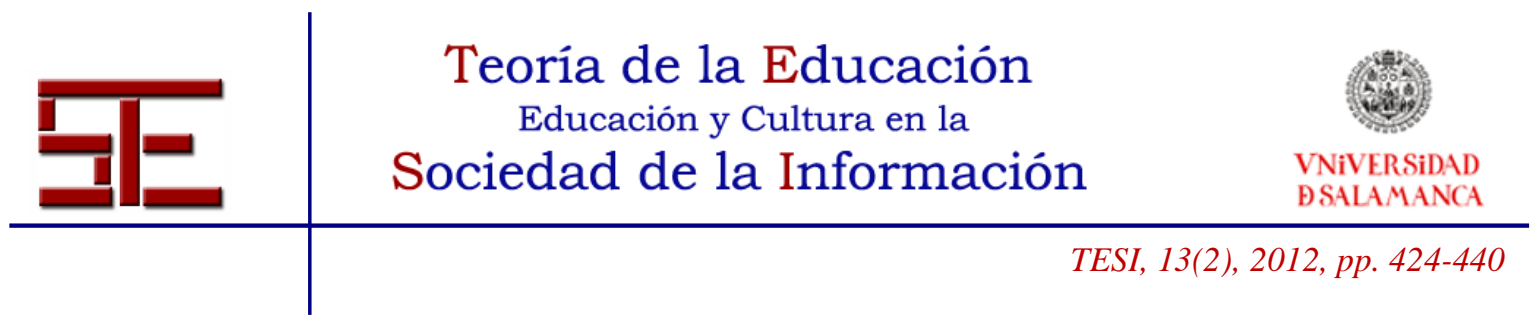

por lo que los docentes tienen una enorme labor en relación a la promoción de este tipo de pensamiento, pues hay cerca de 500 estudiantes, de los cuales 350 hablan lenguas indígenas (totonaco, zoque-popoluca, zapoteco, otomí, huasteco, tepehua, chinanteco) y 227 sólo hablan castellano.

La Universidad Autónoma del Estado de Hidalgo UAEH, en donde labora la autora como docente, también ha mejorado su Modelo Educativo pues en los últimos años se ha capacitado a los docentes de los diversos institutos y Escuelas Superiores dando a conocer las propuestas del nuevo Modelo Educativo Integral Basado en Competencias, que tiene como finalidad que sus egresados respondan a las exigencias del mundo globalizado con una adecuada formación en sus aprendizajes, para lo cual es indispensable la promoción de las competencias genéricas que asume la UAEH, las cuales son siete: formación, liderazgo colaborativo, comunicación, creatividad, pensamiento crítico, uso de la tecnología y ciudadanía.

De manera que el pensamiento crítico en esta universidad se refiere a la aplicación de un pensamiento crítico y autocrítico que apoye identificar, plantear y resolver problemas por medio de procesos de abstracción, análisis y síntesis, discriminando la información de diversas fuentes que permitan un aprendizaje significativo (Modelo Educativo Integral Basado en Competencias. UAEH, 2009).

\section{CONCLUSIONES.}

Las demandas y recomendaciones de los organismos internacionales y la globalización han permeado en las Universidades mexicanas, lo cual es favorable, pues se ha dejado, aunque no en su totalidad, un viejo paradigma con implicaciones educativas que limitaba: el paradigma conductista, que en su momento de auge fue de gran ayuda para solventar necesidades de la sociedad; sin embargo, también el auge de la masificación invita a retomar paradigmas educativos centrados en las motivaciones intrínsecas de los estudiantes; el pensamiento crítico que conlleve a la autonomía de los propios aprendizajes. Aunque, claro está que las Universidades como instituciones tienen sus normas y sus currículos que han de seguirse, por lo que se puede concluir que, aunque los universitarios sean autónomos en sus aprendizajes, no lo son en su totalidad, pues siempre esa autonomía se encontrará subsumida por el currículum, esto en vez de mirarlo como una desventaja que limita al estudiante puede verse como una guía que otorga dirección para saber hacia dónde dirigirse.

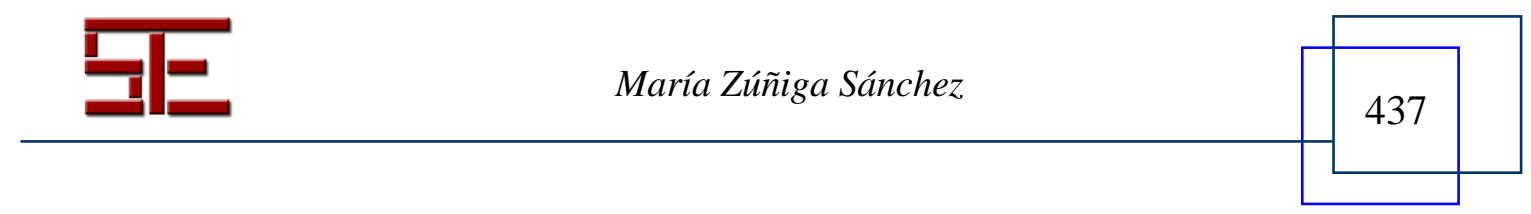




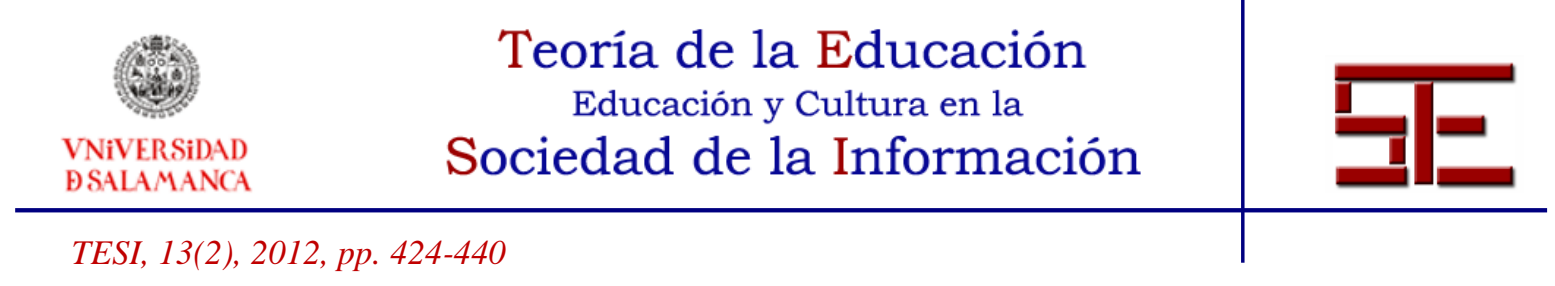

Los universitarios del siglo XXI son invitados a tomar decisiones, a emanciparse y a tomar conciencia de sus potencialidades para participar en la vida social, familiar, económica y política del país. El pensamiento crítico proporciona herramientas indispensables para conseguir lo anterior.

La Universidad Autónoma de Nuevo León, la Universidad Veracruzana y la Universidad Autónoma del Estado de Hidalgo dan cuenta del progreso de desarrollo en la autonomía y pensamiento crítico de sus estudiantes, que están rindiendo frutos en la sociedad mexicana.

Los pilares de la educación que dicta la UNESCO son tomados en cuenta por las universidades mexicanas, especialmente nos enfocamos en Aprender a ser para que la propia personalidad esté en condiciones de obrar con creciente capacidad de autonomía, de juicio y de responsabilidad personal.

Los profesores universitarios tienen el compromiso de fomentar el aprendizaje, la autonomía y el pensamiento crítico en los estudiantes.

\section{0.- BIBLIOGRAFÍA}

Apple, M. W. \& Beane, J. A. (1997). Escuelas democráticas. Madrid: Morata.

Referencias

Alcántara, A. (2006). Tendencias Mundiales en Educación Superior. El papel de los organismos Multilaterales. Revista Interacción. Facultad de Educación UFG. 2. Jan/Jun.

Basañez, M. (2011). Mesa de debate Educación y valores para el desarrollo. En Encuentro Educación y valores para la convivencia en el siglo XXI. México.

Castellano, M. H. (2007). El pensamiento crítico en la escuela. Buenos Aires, Argentina: Prometeo libros.

Consejo de Especialistas (2006). Educación, ciencia y tecnología, y productividad del sistema económico. En Los retos de México en el futuro de la Educación. (pp. 55-56). México: SEP.

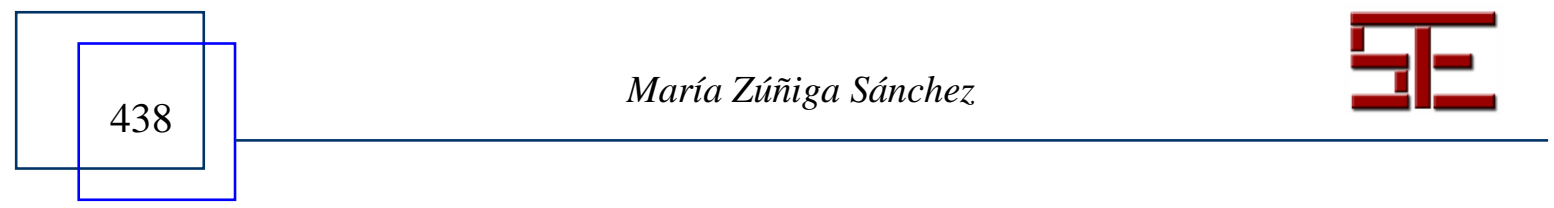




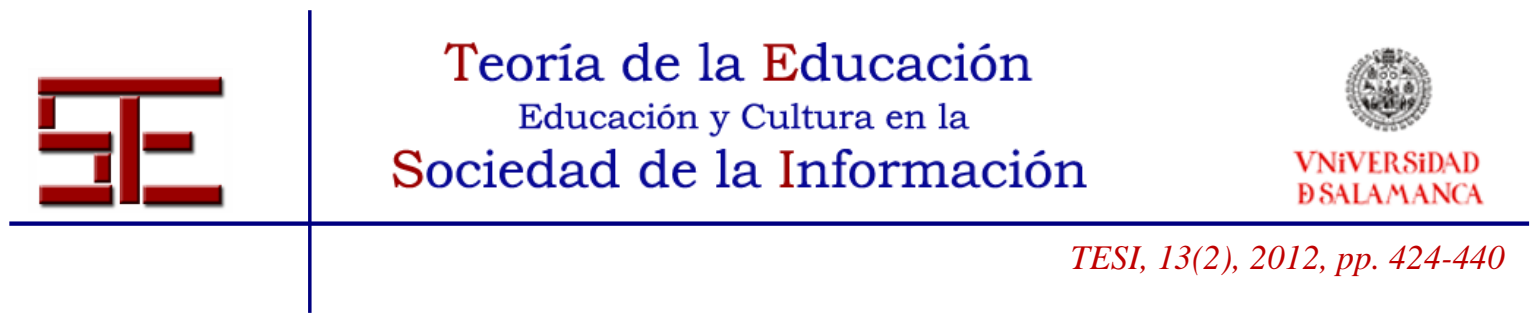

Delors, J. (1999). La educación encierra un tesoro. Informe a la UNESCO de la Comisión Internacional sobre la Educación para el siglo XXI. Ediciones UNESCO.

Díaz, B. F. y Hernández, R. G. (2010). Estrategias docentes para un aprendizaje significativo. México: MC. Graw Hill.

Dietz, G. y Mateos, C. L.S. (2010). Entre culturas, entre saberes, entre poderes: La etnografía reflexiva en el acompañamiento de procesos de interculturalidad educativa (pp. 1-17). En Xochitl Leyva et al.

Galimberti, U. (2006). Diccionario de psicología. México: Siglo XXI.

Gutiérrez, S. (2007). La filosofía del siglo XX. En Historia de las doctrinas filosóficas. México: Esfinge.

Hernández, R. (2006). Paradigmas en psicología de la educación. México: Paídos.

Luengo, G. E. (1993). Tendencias de la Educación Superior en México (pp. 4-18): Una lectura desde la perspectiva de la complejidad.Trabajo elaborado para el Seminario sobre Reformas de la Educación Superior en América Latina y el Caribe, realizado el 5 y 6 de junio del 2003, en Bogotá, Colombia, bajo los auspicios del Instituto Internacional de la UNESCO para la Educación Superior en América Latina y el Caribe (IESACC) y la Asociación Colombiana de Universidades (ASCUN).

Modelo Educativo.(2009). Modelo Educativo Integral Basado en Competencias. México: UAEH.

Navarro, Z, A. \& Rendón, G. (2004). El programa de reflexión universitaria o el diálogo fe y cultura aplicado al currículum universitario. En Las fronteras del diálogo fe y cultura. Una perspectiva transdisciplinar desde la UIA. C. Mendoza (Coord). México: Universidad Iberoamericana A.C.

Rugarcía, T. A. (1997). La formación de ingenieros. Puebla. México: Universidad Iberoamericana.

Saenz, L. K., Galán, W. J. L \& Luna O. H. A. (2003). INNOVACIÓN. Reflexiones sobre la Educación Superior México. Centro de Estudios Sobre la Universidad, Centro de

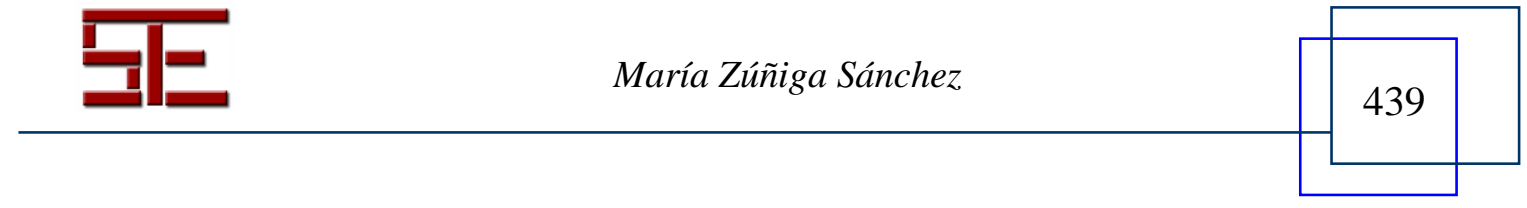




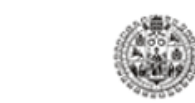

VNIVERSIDAD

DSALAMANCA

TESI, 13(2), 2012, pp. 424-440
Teoria de la Educación

Educación y Cultura en la

Innovación y Competitividad de la Universidad Autónoma de Nuevo León. México: Universidad Autónoma de Nuevo León.

UNESCO (2005) Hacia las sociedades del conocimiento. Ediciones UNESCO. http://unesdoc.unesco.org/images/0014/001419/141908s.pdf.

Para citar el presente artículo puede utilizar la siguiente referencia:

Zúñiga Sánchez, M. (2012). Los estudiantes universitarios del siglo XXI en México: de la pasividad a la autonomía y al pensamiento crítico. Revista Teoría de la Educación: Educación y Cultura en la Sociedad de la Información. 13(2), 424-440 [Fecha de consulta: $\mathrm{dd} / \mathrm{mm} / \mathrm{aaaa}]$.

http://campus.usal.es/ revistas_trabajo/index.php/revistatesi/article/view/9021/9265 